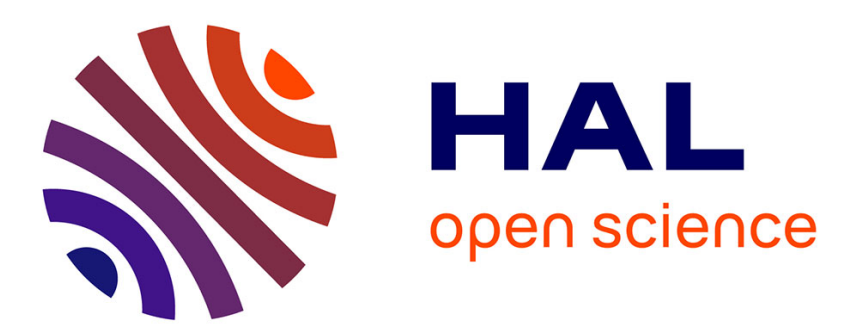

\title{
Sharp interpolation inequalities on the sphere: new methods and consequences
}

Jean Dolbeault, Maria J. Esteban, Michal Kowalczyk, Michael Loss

\section{To cite this version:}

Jean Dolbeault, Maria J. Esteban, Michal Kowalczyk, Michael Loss. Sharp interpolation inequalities on the sphere: new methods and consequences. Chinese Annals of Mathematics - Series B, 2013, 34 (1), pp.99-112. 10.1007/s11401-012-0756-6 . hal-00739140

\section{HAL Id: hal-00739140 https://hal.science/hal-00739140}

Submitted on 5 Oct 2012

HAL is a multi-disciplinary open access archive for the deposit and dissemination of scientific research documents, whether they are published or not. The documents may come from teaching and research institutions in France or abroad, or from public or private research centers.
L'archive ouverte pluridisciplinaire HAL, est destinée au dépôt et à la diffusion de documents scientifiques de niveau recherche, publiés ou non, émanant des établissements d'enseignement et de recherche français ou étrangers, des laboratoires publics ou privés. 


\title{
SHARP INTERPOLATION INEQUALITIES ON THE SPHERE : NEW METHODS AND CONSEQUENCES
}

\author{
JEAN DOLBEAULT, MARIA J. ESTEBAN, MICHAL KOWALCZYK, AND MICHAEL LOSS
}

\begin{abstract}
These notes are devoted to various considerations on a family of sharp interpolation inequalities on the sphere, which in dimension two and higher interpolate between Poincaré, logarithmic Sobolev and critical Sobolev (Onofri in dimension two) inequalities. We emphasize the connexion between optimal constants and spectral properties of the Laplace-Beltrami operator on the sphere. We shall address a series of related observations and give proofs based on symmetrization and the ultraspherical setting.
\end{abstract}

\section{Introduction}

The following interpolation inequality holds on the sphere:

$$
\frac{p-2}{d} \int_{\mathbb{S}^{d}}|\nabla u|^{2} d \mu+\int_{\mathbb{S}^{d}}|u|^{2} d \mu \geq\left(\int_{\mathbb{S}^{d}}|u|^{p} d \mu\right)^{2 / p} \quad \forall u \in \mathrm{H}^{1}\left(\mathbb{S}^{d}, d \mu\right)
$$

for any $p \in\left(2,2^{*}\right]$ with $2^{*}=2 d /(d-2)$ if $d \geq 3$ and for any $p \in(2, \infty)$ if $d=2$. In (1), $d \mu$ is the uniform probability measure on the $d$-dimensional sphere, that is, the measure induced by Lebesgue's measure on $\mathbb{S}^{d} \subset \mathbb{R}^{d+1}$, up to a normalization factor such that $\mu\left(\mathbb{S}^{d}\right)=1$.

Such an inequality has been established by M.-F. Bidaut-Véron and L. Véron in [22] in the more general context of compact manifolds with uniformly positive Ricci curvature. Their method is based on the Bochner-Lichnerowicz-Weitzenböck formula and the study of the set of solutions of an elliptic equation which is seen as a bifurcation problem and contains the Euler-Lagrange equation associated to the optimality case in (1). Later, in [13], W. Beckner gave an alternative proof based on Legendre's duality, on the Funk-Hecke formula, which has been proved in [28, 32], and on the expression of some optimal constants found by E. Lieb in 34. D. Bakry, A. Bentaleb and S. Fahlaoui in a series of papers based on the carré du champ method and mostly devoted to the ultraspherical operator have shown a result which turns out to give yet another proof, which is anyway very close to the method of 22. Their computations allow to slightly extend the range of the parameter $p$ : see $[7,8,14,15,16,17,18,19,20$, and 35,38 , for earlier related works.

In all computations based on the Bochner-Lichnerowicz-Weitzenböck formula, the choice of exponents in the computations appears somewhat mysterious. The seed for such computations can be found in 29]. Our purpose is on one hand to give alternative proofs, at least for some ranges of the parameter $p$, which do not rely on such a very technical choice. On the other hand, we also simplify the existing proofs (see Section 3.2.

Inequality (1) is remarkable for several reasons:

(1) It is optimal in the sense that 1 is the optimal constant. By Hölder's inequality, we know that $\|u\|_{\mathrm{L}^{2}\left(\mathbb{S}^{d}\right)} \leq\|u\|_{\mathrm{L}^{p}\left(\mathbb{S}^{d}\right)}$ so that the equality case can only be achieved by functions which are constant a.e. Of course, the main issue is to prove that the $(p-2) / d$ constant is optimal, which is one of the classical issues of the so-called $A-B$ problem, for which we primarily refer to [31.

(2) If $d \geq 3$, the case $p=2^{*}$ corresponds to Sobolev's inequality. Using the stereographic projection as in 34, we easily recover Sobolev's inequality in the euclidean space $\mathbb{R}^{d}$ with optimal constant and obtain a simple characterization of the extremal functions found by T. Aubin and G. Talenti: see [5, 36, 37.

Date: October 5, 2012.

Key words and phrases. Sobolev inequality; interpolation; Gagliardo-Nirenberg inequalities; logarithmic Sobolev inequality; heat equation

Mathematics Subject Classification (2010). 26D10; 46E35; 58E35. 
(3) In the limit $p \rightarrow 2$, one obtains the logarithmic Sobolev inequality on the sphere, while by taking $p \rightarrow \infty$ if $d=2$, one recovers Onofri's inequality; see [26] and Corollary 3 below.

Exponents are not restricted to $p>2$. Consider indeed the functional

$$
\mathcal{Q}_{p}[u]:=\frac{p-2}{d} \frac{\int_{\mathbb{S}^{d}}|\nabla u|^{2} d \mu}{\left(\int_{\mathbb{S}^{d}}|u|^{p} d \mu\right)^{2 / p}-\int_{\mathbb{S}^{d}}|u|^{2} d \mu}
$$

for $p \in[1,2) \cup\left(2,2^{*}\right]$ if $d \geq 3$ or $p \in[1,2) \cup(2, \infty)$ if $d=2$, and

$$
\mathcal{Q}_{2}[u]:=\frac{1}{d} \frac{\int_{\mathbb{S}^{d}}|\nabla u|^{2} d \mu}{\int_{\mathbb{S}^{d}}|u|^{2} \log \left(|u|^{2} / \int_{\mathbb{S}^{d}}|u|^{2} d \mu\right) d \mu}
$$

for any $d \geq 1$. Because $d \mu$ is a probability measure, $\left(\int_{\mathbb{S}^{d}}|u|^{p} d \mu\right)^{2 / p}-\int_{\mathbb{S}^{d}}|u|^{2} d \mu$ is nonnegative if $p>2$, nonpositive if $p \in[1,2)$, and equal to zero if and only if $u$ is constant a.e. Denote by $\mathcal{A}$ the $\operatorname{set}$ of $\mathrm{H}^{1}\left(\mathbb{S}^{d}, d \mu\right)$ functions which are not a.e. constant and consider the infimum

$$
\mathcal{I}_{p}:=\inf _{u \in \mathcal{A}} \mathcal{Q}_{p}[u]
$$

With these notations, we can state a slightly more general result than the one of (1), which goes as follows and also covers the range $p \in[1,2]$.

Theorem 1. With the above notations, $\mathcal{I}_{p}=1$ for any $p \in\left[1,2^{*}\right]$ if $d \geq 3$, or any $p \in[1, \infty)$ if $d=1,2$.

As already explained above, in the case $\left(2,2^{*}\right]$ the above theorem was proved first in [22, Corollary 6.2], and then in [13] using previous results by E. Lieb in [34] and the Funk-Hecke formula (see [28, 32]). The case $p=2$ was covered in $\left[13\right.$. The whole range $p \in\left[1,2^{*}\right]$ was covered in the case of the ultraspherical operator in [19, 20]. Here we give alternative proofs for various ranges of $p$, which are less technical, and interesting by themselves, as well as some extensions.

Notice that the case $p=1$ can be written as

$$
\int_{\mathbb{S}^{d}}|\nabla u|^{2} d \mu \geq d\left[\int_{\mathbb{S}^{d}}|u|^{2} d \mu-\left(\int_{\mathbb{S}^{d}}|u| d \mu\right)^{2}\right] \quad \forall u \in \mathrm{H}^{1}\left(\mathbb{S}^{d}, d \mu\right),
$$

which is equivalent to the usual Poincaré inequality

$$
\int_{\mathbb{S}^{d}}|\nabla u|^{2} d \mu \geq d \int_{\mathbb{S}^{d}}|u-\bar{u}|^{2} d \mu \quad \forall u \in \mathrm{H}^{1}\left(\mathbb{S}^{d}, d \mu\right) \quad \text { with } \quad \bar{u}=\int_{\mathbb{S}^{d}} u d \mu .
$$

See Remark 4 for more details. The case $p=2$ provides the logarithmic Sobolev inequality on the sphere. It holds as consequence of the inequality for $p \neq 2$ (see Corollary 2).

For $p \neq 2$, the existence of a minimizer of

$$
u \mapsto \int_{\mathbb{S}^{d}}|\nabla u|^{2} d \mu+\frac{d \mathcal{I}_{p}}{p-2}\left[\|u\|_{\mathrm{L}^{2}\left(\mathbb{S}^{d}\right)}^{2}-\|u\|_{\mathrm{L}^{p}\left(\mathbb{S}^{d}\right)}^{2}\right]
$$

in $\left\{u \in \mathrm{H}^{1}\left(\mathbb{S}^{d}, d \mu\right): \int_{\mathbb{S}^{d}}|u|^{p} d \mu=1\right\}$ is easily achieved by variational methods and will be taken for granted. Compactness for either $p \in[1,2)$ or $2<p<2^{*}$ is indeed classical, while the case $p=2^{*}, d \geq 3$ can be studied by concentration-compactness methods. If a function $u \in \mathrm{H}^{1}\left(\mathbb{S}^{d}, d \mu\right)$ is optimal for (1) with $p \neq 2$, then it is solves the Euler-Lagrange equation

$$
-\Delta_{\mathbb{S}^{d}} u=\frac{d \mathcal{I}_{p}}{p-2}\left[\|u\|_{\mathrm{L}^{p}\left(\mathbb{S}^{d}\right)}^{2-p} u^{p-1}-u\right]
$$

where $\Delta_{\mathbb{S}^{d}}$ denotes the Laplace-Beltrami operator on the sphere $\mathbb{S}^{d}$.

In any case, it is possible to normalize the $\mathrm{L}^{p}\left(\mathbb{S}^{d}\right)$-norm of $u$ to 1 without restriction because of the zero homogeneity of $\mathcal{Q}_{p}$. It turns out that the optimality case is achieved by the constant function, with value $u \equiv 1$ if we assume $\int_{\mathbb{S}^{d}}|u|^{p} d \mu=1$, in which case the inequality degenerates because both sides are equal to 0 . This explains why the dimension $d$ shows up here: the sequence $\left(u_{n}\right)_{n \in \mathbb{N}}$ such that

$$
u_{n}(x)=1+\frac{1}{n} v(x)
$$


with $v \in \mathrm{H}^{1}\left(\mathbb{S}^{d}, d \mu\right)$ such that $\int_{\mathbb{S}^{d}} v d \mu=0$ is indeed minimizing if and only if

$$
\int_{\mathbb{S}^{d}}|\nabla v|^{2} d \mu \geq d \int_{\mathbb{S}^{d}}|v|^{2} d \mu
$$

and the equality case is achieved if $v$ is an optimal function for the above Poincaré inequality, i.e. a function associated to the first non-zero eigenvalue of the Laplace-Beltrami operator $-\Delta_{\mathbb{S}^{d}}$ on the sphere $\mathbb{S}^{d}$. Up to a rotation, this means

$$
v(\xi)=\xi_{d} \quad \forall \xi=\left(\xi_{0}, \xi_{1}, \ldots \xi_{d}\right) \in \mathbb{S}^{d} \subset \mathbb{R}^{d+1}
$$

since $-\Delta_{\mathbb{S}^{d}} v=d v$. Recall that the corresponding eigenspace of $-\Delta_{\mathbb{S}^{d}}$ is $d$ dimensional and generated by the composition of $v$ with an arbitrary rotation.

1.1. The logarithmic Sobolev inequality. As a first classical consequence of (2), we have a logarithmic Sobolev inequality. This result is rather classical; related forms of the result can be found for instance in [10] or in 4].

Corollary 2. Let $d \geq 1$. For any $u \in \mathrm{H}^{1}\left(\mathbb{S}^{d}, d \mu\right) \backslash\{0\}$, we have

$$
\int_{\mathbb{S}^{d}}|u|^{2} \log \left(\frac{|u|^{2}}{\int_{\mathbb{S}^{d}}|u|^{2} d \mu}\right) d \mu \leq \frac{2}{d} \int_{\mathbb{S}^{d}}|\nabla u|^{2} d \mu .
$$

Moreover, the constant $\frac{2}{d}$ is sharp.

Proof. The inequality is achieved by taking the limit as $p \rightarrow 2$ in (2). To see that the constant $\frac{2}{d}$ is sharp, we can observe that

$$
\lim _{\varepsilon \rightarrow 0} \int_{\mathbb{S}^{d}}|1+\varepsilon v|^{2} \log \left(\frac{|1+\varepsilon v|^{2}}{\int_{\mathbb{S}^{d}}|1+\varepsilon v|^{2} d \mu}\right) d \mu=2 \int_{\mathbb{S}^{d}}|v-\bar{v}|^{2} d \mu
$$

with $\bar{v}=\int_{\mathbb{S}^{d}} v d \mu$. The result follows by taking $v(\xi)=\xi_{d}$.

\section{Extensions}

2.1. Onofri's inequality. In case of dimension $d=2$, (1) holds for any $p>2$ and we recover Onofri's inequality by taking the limit $p \rightarrow \infty$. This result is standard in the literature: see for instance [13]. For completeness, let us give a statement and a short proof.

Corollary 3. Let $d=1$ or $d=2$. For any $v \in \mathrm{H}^{1}\left(\mathbb{S}^{d}, d \mu\right)$, we have

$$
\int_{\mathbb{S}^{d}} e^{v-\bar{v}} d \mu \leq e^{\frac{1}{2 d} \int_{\mathbb{S}^{d}}|\nabla v|^{2} d \mu}
$$

where $\bar{v}=\int_{\mathbb{S} d} v d \mu$ is the average of $v$. Moreover, the constant $\frac{1}{2 d}$ in the right hand side is sharp.

Proof. In dimension $d=1$ or $d=2$, Inequality (1) holds for any $p>2$. Take $u=1+v / p$ and consider the limit as $p \rightarrow \infty$. We observe that

$$
\int_{\mathbb{S}^{d}}|\nabla u|^{2} d \mu=\frac{1}{p^{2}} \int_{\mathbb{S}^{d}}|\nabla v|^{2} d \mu \quad \text { and } \quad \lim _{p \rightarrow \infty} \int_{\mathbb{S}^{d}}|u|^{p} d \mu=\int_{\mathbb{S}^{d}} e^{v} d \mu
$$

so that

$$
\left(\int_{\mathbb{S}^{d}}|u|^{p} d \mu\right)^{2 / p}-1 \sim \frac{2}{p} \log \left(\int_{\mathbb{S}^{d}} e^{v} d \mu\right) \quad \text { and } \quad \int_{\mathbb{S}^{d}}|u|^{2} d \mu-1 \sim \frac{2}{p} \int_{\mathbb{S}^{d}} v d \mu .
$$

The conclusion holds by passing to the limit $p \rightarrow \infty$ in Inequality (1). Optimality is once more achieved by considering $v=\varepsilon v_{1}, v_{1}(\xi)=\xi_{d}, d=1$ and Taylor expanding both sides of the inequality in terms of $\varepsilon>0$, small. Notice indeed that $-\Delta_{\mathbb{S}^{d}} v_{1}=\lambda_{1} v_{1}$ with $\lambda_{1}=d$, so that

$$
\|\nabla u\|_{\mathrm{L}^{2}\left(\mathbb{S}^{d}\right)}^{2}=\varepsilon^{2}\left\|\nabla v_{1}\right\|_{\mathrm{L}^{2}\left(\mathbb{S}^{d}\right)}^{2}=\varepsilon^{2} d\left\|v_{1}\right\|_{\mathrm{L}^{2}\left(\mathbb{S}^{d}\right)}^{2},
$$

$\int_{\mathbb{S}^{d}} v_{1} d \mu=\bar{v}_{1}=0$, and

$$
\int_{\mathbb{S}^{d}} e^{v-\bar{v}} d \mu-1 \sim \frac{\varepsilon^{2}}{2} \int_{\mathbb{S}^{d}}|v-\bar{v}|^{2} d \mu=\frac{1}{2} \varepsilon^{2}\left\|v_{1}\right\|_{\mathrm{L}^{2}\left(\mathbb{S}^{d}\right)}^{2} .
$$


2.2. Interpolation and a spectral approach for $p \in(1,2)$. In 11, W. Beckner gave a method to prove interpolation inequalities between logarithmic Sobolev and Poincaré inequalities in case of a Gaussian measure. Here we shall prove that the method extends to the case of the sphere and therefore provides another family of interpolating inequalities, in a new range: $p \in[1,2)$, again with optimal constants. For further considerations on inequalities that interpolate between Poincaré and logarithmic Sobolev inequalities, we refer to [3, 1, 2, 10, 9, 23, 24, 27, 33] and references therein.

Our purpose is to extend (1) written as

$$
\frac{1}{d} \int_{\mathbb{S}^{d}}|\nabla u|^{2} d \mu \geq \frac{\left(\int_{\mathbb{S}^{d}}|u|^{p} d \mu\right)^{2 / p}-\int_{\mathbb{S}^{d}}|u|^{2} d \mu}{p-2} \quad \forall u \in \mathrm{H}^{1}\left(\mathbb{S}^{d}, d \mu\right)
$$

to the case $p \in[1,2)$. Let us start with a remark.

Remark 4. At least for any nonnegative function $v$, using the fact that $\mu$ is a probability measure on $\mathbb{S}^{d}$, we may notice that

$$
\int_{\mathbb{S}^{d}}|v-\bar{v}|^{2} d \mu=\int_{\mathbb{S}^{d}}|v|^{2} d \mu-\left(\int_{\mathbb{S}^{d}} v d \mu\right)^{2}
$$

can be rewritten as

$$
\int_{\mathbb{S}^{d}}|v-\bar{v}|^{2} d \mu=\frac{\int_{\mathbb{S}^{d}}|v|^{2} d \mu-\left(\int_{\mathbb{S}^{d}}|v|^{p} d \mu\right)^{2 / p}}{2-p},
$$

for $p=1$, hence extending (1) to the case $q=1$. However, as already noticed for instance in [1], the inequality

$$
\int_{\mathbb{S}^{d}}|v|^{2} d \mu-\left(\int_{\mathbb{S}^{d}}|v| d \mu\right)^{2} \leq \frac{1}{d} \int_{\mathbb{S}^{d}}|\nabla v|^{2} d \mu
$$

also means that, for any $c \in \mathbb{R}$,

$$
\int_{\mathbb{S}^{d}}|v+c|^{2} d \mu-\left(\int_{\mathbb{S}^{d}}|v+c| d \mu\right)^{2} \leq \frac{1}{d} \int_{\mathbb{S}^{d}}|\nabla v|^{2} d \mu .
$$

If $v$ is bounded from below a.e. with respect to $\mu$ and $c>-\operatorname{infess}_{\mu} v$, so that $v+c>0 \mu$ a.e., the left hand-side is

$$
\int_{\mathbb{S}^{d}}|v+c|^{2} d \mu-\left(\int_{\mathbb{S}^{d}}|v+c| d \mu\right)^{2}=c^{2}+2 c \int_{\mathbb{S}^{d}} v d \mu+\int_{\mathbb{S}^{d}}|v|^{2} d \mu-\left(c+\int_{\mathbb{S}^{d}} v d \mu\right)^{2}=\int_{\mathbb{S}^{d}}|v-\bar{v}|^{2} d \mu,
$$

so that the inequality is the usual Poincaré inequality. By density, we recover that (4) written for $p=1$ exactly amounts to Poincaré's inequality written not only for $|v|$, but also for any $v \in \mathrm{H}^{1}\left(\mathbb{S}^{d}, d \mu\right)$.

Next, using the method introduced by W. Beckner in [11 in case of a Gaussian measure, we are in position to prove (4) for any $p \in(1,2)$, knowing that the inequality holds for $p=1$ and $p=2$.

Proposition 5. Inequality (4) holds for any $p \in(1,2)$ and any $d \geq 1$. Moreover $d$ is the optimal constant.

Proof. Optimality can be checked by Taylor expanding $u=1+\varepsilon v$ at order two in terms of $\varepsilon>0$ as in the case $p=2$ (logarithmic Sobolev inequality). To establish the inequality itself, we may proceed in two steps.

$1^{\text {st }}$ step: Nelson's hypercontractivity result. Although the result can be established by direct methods, we follow here the strategy of Gross in [30, which proves the equivalence of the optimal hypercontractivity result and the optimal logarithmic Sobolev inequality.

Consider the heat equation of $\mathbb{S}^{d}$, namely

$$
\frac{\partial f}{\partial t}=\Delta_{\mathbb{S}^{d}} f
$$


with initial datum $f(t=0, \cdot)=u \in L^{2 / p}\left(\mathbb{S}^{d}\right)$, for some $p \in(1,2]$, and let $F(t):=\|f(t, \cdot)\|_{\mathrm{L}^{p(t)}\left(\mathbb{S}^{d}\right)}$. The key computation goes as follows.

$$
\begin{aligned}
\frac{F^{\prime}}{F}=\frac{d}{d t} \log F(t)=\frac{d}{d t}\left[\frac{1}{p(t)} \log \left(\int_{\mathbb{S}^{d}}|f(t, \cdot)|^{p(t)} d \mu\right)\right] \\
=\frac{p^{\prime}}{p^{2} F^{p}}\left[\int_{\mathbb{S}^{d}} v^{2} \log \left(\frac{v^{2}}{\int_{\mathbb{S}^{d}} v^{2} d \mu}\right) d \mu+4 \frac{p-1}{p^{\prime}} \int_{\mathbb{S}^{d}}|\nabla v|^{2} d \mu\right]
\end{aligned}
$$

with $v:=|f|^{p(t) / 2}$. Assuming that $4 \frac{p-1}{p^{\prime}}=\frac{2}{d}$, that is

we find that

$$
\frac{p^{\prime}}{p-1}=2 d
$$

$$
\log \left(\frac{p(t)-1}{p-1}\right)=2 d t
$$

if we require that $p(0)=p<2$. Let $t_{*}>0$ be such that $p\left(t_{*}\right)=2$. As a consequence of the above computation, we have

$$
\left\|f\left(t_{*}, \cdot\right)\right\|_{\mathrm{L}^{2}\left(\mathbb{S}^{d}\right)} \leq\|u\|_{\mathrm{L}^{2 / p}\left(\mathbb{S}^{d}\right)} \quad \text { if } \quad \frac{1}{p-1}=e^{2 d t_{*}} .
$$

$2^{\text {nd }}$ step: Spectral decomposition. Let $u=\sum_{k \in \mathbb{N}} u_{k}$ be a decomposition of the initial datum on the eigenspaces of $-\Delta_{\mathbb{S}^{d}}$ and denote by $\lambda_{k}=k(d+k-1)$ the ordered sequence of the eigenvalues: $-\Delta_{\mathbb{S}^{d}} u_{k}=$ $\lambda_{k} u_{k}$ (see for instance [21]). Let $a_{k}=\left\|u_{k}\right\|_{\mathrm{L}^{2}\left(\mathbb{S}^{d}\right)}^{2}$. As a straightforward consequence of this decomposition, we know that $\|u\|_{\mathrm{L}^{2}\left(\mathbb{S}^{d}\right)}^{2}=\sum_{k \in \mathbb{N}} a_{k},\|\nabla u\|_{\mathrm{L}^{2}\left(\mathbb{S}^{d}\right)}^{2}=\sum_{k \in \mathbb{N}} \lambda_{k} a_{k}$,

$$
\left\|f\left(t_{*}, \cdot\right)\right\|_{\mathrm{L}^{2}\left(\mathbb{S}^{d}\right)}^{2}=\sum_{k \in \mathbb{N}} a_{k} e^{-2 \lambda_{k} t_{*}} .
$$

Using (5), it follows that

$$
\frac{\left(\int_{\mathbb{S}^{d}}|u|^{p} d \mu\right)^{2 / p}-\int_{\mathbb{S}^{d}}|u|^{2} d \mu}{p-2} \leq \frac{\left(\int_{\mathbb{S}^{d}}|u|^{2} d \mu\right)-\int_{\mathbb{S}^{d}}\left|f\left(t_{*}, \cdot\right)\right|^{2} d \mu}{2-p}=\frac{1}{2-p} \sum_{k \in \mathbb{N}^{*}} \lambda_{k} a_{k} \frac{1-e^{-2 \lambda_{k} t_{*}}}{\lambda_{k}} .
$$

Notice that $\lambda_{0}=0$ so that the term corresponding to $k=0$ can be omitted in the series. Since $\lambda \mapsto$ $\frac{1-e^{-2 \lambda t_{*}}}{\lambda}$ is decreasing, we can bound $\frac{1-e^{-2 \lambda_{k} t_{*}}}{\lambda_{k}}$ from above by $\frac{1-e^{-2 \lambda_{1} t_{*}}}{\lambda_{1}}$ for any $k \geq 1$. This proves that

$$
\frac{\left(\int_{\mathbb{S}^{d}}|u|^{p} d \mu\right)^{2 / p}-\int_{\mathbb{S}^{d}}|u|^{2} d \mu}{p-2} \leq \frac{1-e^{-2 \lambda_{1} t_{*}}}{(2-p) \lambda_{1}} \sum_{k \in \mathbb{N}^{*}} \lambda_{k} a_{k}=\frac{1-e^{-2 \lambda_{1} t_{*}}}{(2-p) \lambda_{1}}\|\nabla u\|_{\mathrm{L}^{2}\left(\mathbb{S}^{d}\right)}^{2} .
$$

The conclusion easily follows if we notice that $\lambda_{1}=d$, and $e^{-2 \lambda_{1} t_{*}}=p-1$ so that

$$
\frac{1-e^{-2 \lambda_{1} t_{*}}}{(2-p) \lambda_{1}}=\frac{1}{d}
$$

The optimality of this constant can be checked as in the case $p>2$ by a Taylor expansion of $u=1+\varepsilon v$ at order two in terms of $\varepsilon>0$, small.

\section{Symmetrization AND the Ultraspherical FRAMEWORK}

3.1. A reduction to the ultraspherical framework. We denote by $\left(\xi_{0}, \xi_{1}, \ldots \xi_{d}\right)$ the coordinates of an arbitrary point $\xi \in \mathbb{S}^{d}$, with $\sum_{i=0}^{d}\left|\xi_{i}\right|^{2}=1$. The following symmetry result is kind of folklore in the literature and we can quote [6, 34, 12] for various related results.

Lemma 6. Up to a rotation, any minimizer of (2) depends only on $\xi_{d}$.

Proof. Let $u$ be a minimizer for $\mathcal{Q}_{p}$. By writing $u$ in (1) in spherical coordinates $\theta \in[0, \pi], \varphi_{1}, \varphi_{2}, \ldots$ $\varphi_{d-1} \in[0,2 \pi)$ and using decreasing rearrangements (see for instance [25]), it is not difficult to prove that among optimal functions, there is one which depends only on $\theta$. Moreover, equality in the rearrangement inequality means that $u$ has to depend on only one coordinate, $\xi_{d}=\sin \theta$. 
Let us observe that the problem on the sphere can be reduced to a problem involving the ultraspherical operator:

- Using Lemma 6, we know that (1) is equivalent to

$$
\frac{p-2}{d} \int_{0}^{\pi}\left|v^{\prime}(\theta)\right|^{2} d \sigma+\int_{0}^{\pi}|v(\theta)|^{2} d \sigma \geq\left(\int_{0}^{\pi}|v(\theta)|^{p} d \sigma\right)^{\frac{2}{p}}
$$

for any function $v \in \mathrm{H}^{1}([0, \pi], d \sigma)$, where

$$
d \sigma(\theta):=\frac{(\sin \theta)^{d-1}}{Z_{d}} d \theta \quad \text { with } \quad Z_{d}:=\sqrt{\pi} \frac{\Gamma\left(\frac{d}{2}\right)}{\Gamma\left(\frac{d+1}{2}\right)} .
$$

- The change of variables $x=\cos \theta, v(\theta)=f(x)$ allows to rewrite the inequality as

$$
\frac{p-2}{d} \int_{-1}^{1}\left|f^{\prime}\right|^{2} \nu d \nu_{d}+\int_{-1}^{1}|f|^{2} d \nu_{d} \geq\left(\int_{-1}^{1}|f|^{p} d \nu_{d}\right)^{\frac{2}{p}}
$$

where $d \nu_{d}$ is the probability measure defined by

$$
\nu_{d}(x) d x=d \nu_{d}(x):=Z_{d}^{-1} \nu^{\frac{d}{2}-1} d x \quad \text { with } \quad \nu(x):=1-x^{2}, \quad Z_{d}=\sqrt{\pi} \frac{\Gamma\left(\frac{d}{2}\right)}{\Gamma\left(\frac{d+1}{2}\right)} .
$$

We may also want to prove the result in case $p<2$, to have the counterpart of Theorem 1 in the ultraspherical setting. On $[-1,1]$, consider the probability measure $d \nu_{d}$ and define

$$
\nu(x):=1-x^{2},
$$

so that $d \nu_{d}=Z_{d}^{-1} \nu^{\frac{d}{2}-1} d x$. We consider the space $\mathrm{L}^{2}\left((-1,1), d \nu_{d}\right)$ with scalar product

$$
\left\langle f_{1}, f_{2}\right\rangle=\int_{-1}^{1} f_{1} f_{2} d \nu_{d}
$$

and use the notation

$$
\|f\|_{p}=\left(\int_{-1}^{1} f^{p} d \nu_{d}\right)^{\frac{1}{p}} .
$$

On $\mathrm{L}^{2}\left((-1,1), d \nu_{d}\right)$, we define the self-adjoint ultraspherical operator by

$$
\mathcal{L} f:=\left(1-x^{2}\right) f^{\prime \prime}-d x f^{\prime}=\nu f^{\prime \prime}+\frac{d}{2} \nu^{\prime} f^{\prime}
$$

which satisfies the identity

$$
\left\langle f_{1}, \mathcal{L} f_{2}\right\rangle=-\int_{-1}^{1} f_{1}^{\prime} f_{2}^{\prime} \nu d \nu_{d}
$$

Then the result goes as follows.

Proposition 7. Let $p \in\left[1,2^{*}\right], d \geq 1$. Then we have

$$
-\langle f, \mathcal{L} f\rangle=\int_{-1}^{1}\left|f^{\prime}\right|^{2} \nu d \nu_{d} \geq d \frac{\|f\|_{p}^{2}-\|f\|_{2}^{2}}{p-2} \quad \forall f \in \mathrm{H}^{1}\left([-1,1], d \nu_{d}\right)
$$

if $p \neq 2$, and

$$
-\langle f, \mathcal{L} f\rangle=\frac{d}{2} \int_{-1}^{1}|f|^{2} \log \left(\frac{|f|^{2}}{\|f\|_{2}^{2}}\right) d \nu_{d}
$$

if $p=2$.

We may notice that the proof in 22 requires $d \geq 2$ while the case $d=1$ is also covered in [13. In Bentaleb et al., the restriction $d \geq 2$ has been removed in [20. Our proof is inspired by [22] and [15, 18, but it is a simplification (in the particular case of the ultraspherical operator) in the sense that only integration by parts and elementary estimates are used. 
3.2. A proof of Proposition 7, Let us start with some preliminary observations. The operator $\mathcal{L}$ does not commute with the derivation, but we have the relation

$$
\left[\frac{\partial}{\partial x}, \mathcal{L}\right] u=(\mathcal{L} u)^{\prime}-\mathcal{L} u^{\prime}=-2 x u^{\prime \prime}-d u^{\prime}
$$

As a consequence, we obtain

$$
\langle\mathcal{L} u, \mathcal{L} u\rangle=-\int_{-1}^{1} u^{\prime}(\mathcal{L} u)^{\prime} \nu d \nu_{d}=-\int_{-1}^{1} u^{\prime} \mathcal{L} u^{\prime} \nu d \nu_{d}+\int_{-1}^{1} u^{\prime}\left(2 x u^{\prime \prime}+d u^{\prime}\right) \nu d \nu_{d}
$$

and

$$
\begin{gathered}
\langle\mathcal{L} u, \mathcal{L} u\rangle=\int_{-1}^{1}\left|u^{\prime \prime}\right|^{2} \nu^{2} d \nu_{d}-d\langle u, \mathcal{L} u\rangle \\
\int_{-1}^{1}(\mathcal{L} u)^{2} d \nu_{d}=\langle\mathcal{L} u, \mathcal{L} u\rangle=\int_{-1}^{1}\left|u^{\prime \prime}\right|^{2} \nu^{2} d \nu_{d}+d \int_{-1}^{1}\left|u^{\prime}\right|^{2} \nu d \nu_{d} .
\end{gathered}
$$

On the other hand, a few integrations by parts show that

$$
\left\langle\frac{\left|u^{\prime}\right|^{2}}{u} \nu, \mathcal{L} u\right\rangle=\frac{d}{d+2} \int_{-1}^{1} \frac{\left|u^{\prime}\right|^{4}}{u^{2}} \nu^{2} d \nu_{d}-2 \frac{d-1}{d+2} \int_{-1}^{1} \frac{\left|u^{\prime}\right|^{2} u^{\prime \prime}}{u} \nu^{2} d \nu_{d},
$$

where we have used the fact that $\nu \nu^{\prime} \nu_{d}=\frac{2}{d+2}\left(\nu^{2} \nu_{d}\right)^{\prime}$.

Let $p \in(1,2) \cup\left(2,2^{*}\right)$. In $H^{1}\left([-1,1], d \nu_{d}\right)$, consider now a minimizer $f$ for the functional

$$
f \mapsto \int_{-1}^{1}\left|f^{\prime}\right|^{2} \nu d \nu_{d}-d \frac{\|f\|_{p}^{2}-\|f\|_{2}^{2}}{p-2}=: \mathcal{F}[f]
$$

made of the difference of the two sides in inequality (6). The existence of such a minimizer can be proved by classical minimization and compactness arguments. Up to a multiplication by a constant, $f$ satisfies the Euler-Lagrange equation

$$
-\frac{p-2}{d} \mathcal{L} f+f=f^{p-1} .
$$

Let $\beta$ be a real number to be fixed later and define $u$ such that $f=u^{\beta}$, so that

$$
\mathcal{L} f=\beta u^{\beta-1}\left(\mathcal{L} u+(\beta-1) \frac{\left|u^{\prime}\right|^{2}}{u} \nu\right) .
$$

Then $u$ is a solution to

$$
-\mathcal{L} u-(\beta-1) \frac{\left|u^{\prime}\right|^{2}}{u} \nu+\lambda u=\lambda u^{1+\beta(p-2)} \quad \text { with } \quad \lambda:=\frac{d}{(p-2) \beta} .
$$

If we multiply the equation for $u$ by $\frac{\left|u^{\prime}\right|^{2}}{u} \nu$ and integrate, we get

$$
-\int_{-1}^{1} \mathcal{L} u \frac{\left|u^{\prime}\right|^{2}}{u} \nu d \nu_{d}-(\beta-1) \int_{-1}^{1} \frac{\left|u^{\prime}\right|^{4}}{u^{2}} \nu^{2} d \nu_{d}+\lambda \int_{-1}^{1}\left|u^{\prime}\right|^{2} \nu d \nu_{d}=\lambda \int_{-1}^{1} u^{\beta(p-2)}\left|u^{\prime}\right|^{2} \nu d \nu_{d} .
$$

If we multiply the equation for $u$ by $-\mathcal{L} u$ and integrate, we get

$$
\int_{-1}^{1}(\mathcal{L} u)^{2} d \nu_{d}+(\beta-1) \int_{-1}^{1} \mathcal{L} u \frac{\left|u^{\prime}\right|^{2}}{u} \nu d \nu_{d}+\lambda \int_{-1}^{1}\left|u^{\prime}\right|^{2} \nu d \nu_{d}=(\lambda+d) \int_{-1}^{1} u^{\beta(p-2)}\left|u^{\prime}\right|^{2} \nu d \nu_{d} .
$$

Collecting terms, we have found that

$$
\int_{-1}^{1}(\mathcal{L} u)^{2} d \nu_{d}+\left(\beta+\frac{d}{\lambda}\right) \int_{-1}^{1} \mathcal{L} u \frac{\left|u^{\prime}\right|^{2}}{u} \nu d \nu_{d}+(\beta-1)\left(1+\frac{d}{\lambda}\right) \int_{-1}^{1} \frac{\left|u^{\prime}\right|^{4}}{u^{2}} \nu^{2} d \nu_{d}-d \int_{-1}^{1}\left|u^{\prime}\right|^{2} \nu d \nu_{d}=0 .
$$

Using (7) and (8), we get

$$
\begin{array}{r}
\int_{-1}^{1}\left|u^{\prime \prime}\right|^{2} \nu^{2} d \nu_{d}+\left(\beta+\frac{d}{\lambda}\right)\left[\frac{d}{d+2} \int_{-1}^{1} \frac{\left|u^{\prime}\right|^{4}}{u^{2}} \nu^{2} d \nu_{d}-2 \frac{d-1}{d+2} \int_{-1}^{1} \frac{\left|u^{\prime}\right|^{2} u^{\prime \prime}}{u} \nu^{2} d \nu_{d}\right] \\
+(\beta-1)\left(1+\frac{d}{\lambda}\right) \int_{-1}^{1} \frac{\left|u^{\prime}\right|^{4}}{u^{2}} \nu^{2} d \nu_{d}=0,
\end{array}
$$


that is

$$
\mathrm{a} \int_{-1}^{1}\left|u^{\prime \prime}\right|^{2} \nu^{2} d \nu_{d}+2 \mathrm{~b} \int_{-1}^{1} \frac{\left|u^{\prime}\right|^{2} u^{\prime \prime}}{u} \nu^{2} d \nu_{d}+\mathrm{c} \int_{-1}^{1} \frac{\left|u^{\prime}\right|^{4}}{u^{2}} \nu^{2} d \nu_{d}=0
$$

where

$$
\begin{aligned}
& \mathrm{a}=1, \\
& \mathrm{~b}=-\left(\beta+\frac{d}{\lambda}\right) \frac{d-1}{d+2}, \\
& \mathrm{c}=\left(\beta+\frac{d}{\lambda}\right) \frac{d}{d+2}+(\beta-1)\left(1+\frac{d}{\lambda}\right) .
\end{aligned}
$$

Using $\frac{d}{\lambda}=(p-2) \beta$, we observe that the reduced discriminant

$$
\delta=\mathrm{b}^{2}-\mathrm{ac}<0
$$

can be written as

$$
\delta=A \beta^{2}+B \beta+1 \quad \text { with } \quad A=(p-1)^{2} \frac{(d-1)^{2}}{(d+2)^{2}}-p+2 \quad \text { and } \quad B=p-3-\frac{d(p-1)}{d+2} .
$$

If $p<2^{*}, B^{2}-4 A$ is positive and it is therefore possible to find $\beta$ such that $\delta<0$.

Hence, if $p<2^{*}$, we have shown that $\mathcal{F}[f]$ is positive unless the three integrals $(9)$ are equal to 0 , that is, $u$ is constant. It follows that $\mathcal{F}[f]=0$, which proves 6 if $p \in(1,2) \cup\left(2,2^{*}\right)$. The cases $p=1, p=2$ ( $c f$. Corollary 2) and $p=2^{*}$ can be proved as limit cases. This concludes the proof of Proposition 7 .

\section{A PROOF BASED ON A FLOW IN THE ULTRASPHERICAL SETTING}

Inequality (6) can be rewritten for $g=f^{p}$, i.e. $f=g^{\alpha}$ with $\alpha=1 / p$, as

$$
-\langle f, \mathcal{L} f\rangle=-\left\langle g^{\alpha}, \mathcal{L} g^{\alpha}\right\rangle=: \mathcal{I}[g] \geq d \frac{\|g\|_{1}^{2 \alpha}-\left\|g^{2 \alpha}\right\|_{1}}{p-2}=: \mathcal{F}[g]
$$

4.1. Flow. Consider the flow associated to $\mathcal{L}$, that is

$$
\frac{\partial g}{\partial t}=\mathcal{L} g
$$

and observe that

$$
\frac{d}{d t}\|g\|_{1}=0, \quad \frac{d}{d t}\left\|g^{2 \alpha}\right\|_{1}=-2(p-2)\langle f, \mathcal{L} f\rangle=2(p-2) \int_{-1}^{1}\left|f^{\prime}\right|^{2} \nu d \nu_{d}
$$

which finally gives

$$
\frac{d}{d t} \mathcal{F}[g(t, \cdot)]=-\frac{d}{p-2} \frac{d}{d t}\left\|g^{2 \alpha}\right\|_{1}=-2 d \mathcal{I}[g(t, \cdot)]
$$

4.2. Method. If (6) holds, then

$$
\frac{d}{d t} \mathcal{F}[g(t, \cdot)] \leq-2 d \mathcal{F}[g(t, \cdot)],
$$

thus proving

$$
\mathcal{F}[g(t, \cdot)] \leq \mathcal{F}[g(0, \cdot)] e^{-2 d t} \quad \forall t \geq 0 .
$$

This estimate is actually equivalent to (6) as can be shown by estimating $\frac{d}{d t} \mathcal{F}[g(t, \cdot)]$ at $t=0$.

The method based on the Bakry-Emery approach amounts to establish first that

$$
\frac{d}{d t} \mathcal{I}[g(t, \cdot)] \leq-2 d \mathcal{I}[g(t, \cdot)]
$$

and prove (11) by integrating the estimate on $t \in[0, \infty)$ : since

$$
\frac{d}{d t}(\mathcal{F}[g(t, \cdot)]-\mathcal{I}[g(t, \cdot)]) \geq 0
$$

and $\lim _{t \rightarrow \infty}(\mathcal{F}[g(t, \cdot)]-\mathcal{I}[g(t, \cdot)])=0$, this means that

$$
\mathcal{F}[g(t, \cdot)]-\mathcal{I}[g(t, \cdot)] \leq 0 \quad \forall t \geq 0
$$


which is precisely (6) written for $f(t, \cdot)$ for any $t \geq 0$ and in particular for any initial value $f(0, \cdot)$.

The equation for $g=f^{p}$ can be rewritten in terms of $f$ as

$$
\frac{\partial f}{\partial t}=\mathcal{L} f+(p-1) \frac{\left|f^{\prime}\right|^{2}}{f} \nu .
$$

Hence we have

$$
-\frac{1}{2} \frac{d}{d t} \int_{-1}^{1}\left|f^{\prime}\right|^{2} \nu d \nu_{d}=\frac{1}{2} \frac{d}{d t}\langle f, \mathcal{L} f\rangle=\langle\mathcal{L} f, \mathcal{L} f\rangle+(p-1)\left\langle\frac{\left|f^{\prime}\right|^{2}}{f} \nu, \mathcal{L} f\right\rangle
$$

4.3. An inequality for the Fisher information. Instead of proving (6), we will established the following stronger inequality. For any $p \in\left(2,2^{\sharp}\right]$,

$$
\langle\mathcal{L} f, \mathcal{L} f\rangle+(p-1)\left\langle\frac{\left|f^{\prime}\right|^{2}}{f} \nu, \mathcal{L} f\right\rangle+d\langle f, \mathcal{L} f\rangle \geq 0
$$

Notice that (6) holds under the restriction $p \in\left(2,2^{\sharp}\right]$, which is stronger than $p \in\left(2,2^{*}\right]$. We do not know whether the exponent $2^{\sharp}$ in $(13)$ is sharp or not.

4.4. Proof of (13). Using (7) and (8) with $u=f$, we find that

$$
\begin{aligned}
\frac{d}{d t} \int_{-1}^{1}\left|f^{\prime}\right|^{2} \nu d \nu_{d}+2 d \int_{-1}^{1} \mid & \left.f^{\prime}\right|^{2} \nu d \nu_{d} \\
& =-2 \int_{-1}^{1}\left(\left|f^{\prime \prime}\right|^{2}+(p-1) \frac{d}{d+2} \frac{\left|f^{\prime}\right|^{4}}{f^{2}}-2(p-1) \frac{d-1}{d+2} \frac{\left|f^{\prime}\right|^{2} f^{\prime \prime}}{f}\right) \nu^{2} d \nu_{d} .
\end{aligned}
$$

The right hand side is nonpositive if

$$
\left|f^{\prime \prime}\right|^{2}+(p-1) \frac{d}{d+2} \frac{\left|f^{\prime}\right|^{4}}{f^{2}}-2(p-1) \frac{d-1}{d+2} \frac{\left|f^{\prime}\right|^{2} f^{\prime \prime}}{f}
$$

is pointwise nonnegative, which is granted if

$$
\left[(p-1) \frac{d-1}{d+2}\right]^{2} \leq(p-1) \frac{d}{d+2},
$$

a condition which is exactly equivalent to $p \leq 2^{\sharp}$.

4.5. An improved inequality. For any $p \in\left(2,2^{\sharp}\right)$, we can write that

$$
\begin{aligned}
&\left|f^{\prime \prime}\right|^{2}+(p-1) \frac{d}{d+2} \frac{\left|f^{\prime}\right|^{4}}{f^{2}}-2(p-1) \frac{d-1}{d+2} \frac{\left|f^{\prime}\right|^{2} f^{\prime \prime}}{f} \\
&=\alpha\left|f^{\prime \prime}\right|^{2}+\frac{p-1}{d+2}\left|\frac{d-1}{\sqrt{d}} f^{\prime \prime}-\sqrt{d} \frac{\left|f^{\prime}\right|^{2}}{f}\right|^{2} \geq \alpha\left|f^{\prime \prime}\right|^{2}
\end{aligned}
$$

where

$$
\alpha:=1-(p-1) \frac{(d-1)^{2}}{d(d+2)}
$$

is positive. Now, using the Poincaré inequality

$$
\int_{-1}^{1}\left|f^{\prime \prime}\right|^{2} d \nu_{d+4} \geq(d+2) \int_{-1}^{1}\left|f^{\prime}-\overline{f^{\prime}}\right|^{2} d \nu_{d+2}
$$

where

$$
\overline{f^{\prime}}:=\int_{-1}^{1} f^{\prime} d \nu_{d+2}=-d \int_{-1}^{1} x f d \nu_{d},
$$

we obtain an improved form of 13 , namely

$$
\langle\mathcal{L} f, \mathcal{L} f\rangle+(p-1)\left\langle\frac{\left|f^{\prime}\right|^{2}}{f} \nu, \mathcal{L} f\right\rangle+[d+\alpha(d+2)]\langle f, \mathcal{L} f\rangle \geq 0
$$




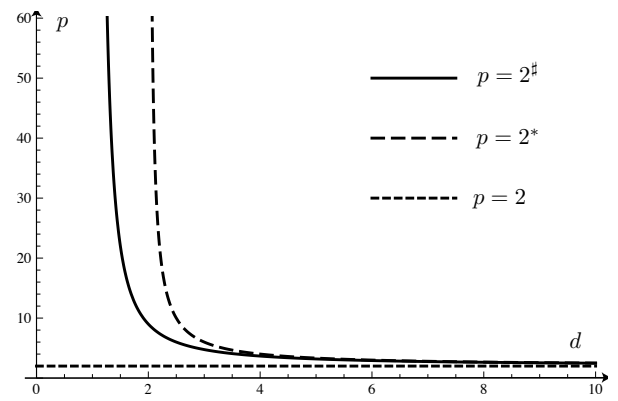

Figure 1. Plot of $d \mapsto 2^{\sharp}=\frac{2 d^{2}+1}{(d-1)^{2}}$ and $d \mapsto 2^{*}=\frac{2 d}{d-2}$.

if we can guarantee that $\overline{f^{\prime}} \equiv 0$ along the evolution determined by $[10$. This is the case if assume that $f(x)=f(-x)$ for any $x \in[-1,1]$. Under this condition, we find that

$$
\int_{-1}^{1}\left|f^{\prime}\right|^{2} \nu d \nu_{d} \geq[d+\alpha(d+2)] \frac{\|f\|_{p}^{2}-\|f\|_{2}^{2}}{p-2} .
$$

As a consequence, we also have

$$
\int_{\mathbb{S}^{d}}|\nabla u|^{2} d \mu+\int_{\mathbb{S}^{d}}|u|^{2} d \mu \geq \frac{d+\alpha(d+2)}{p-2}\left(\int_{\mathbb{S}^{d}}|u|^{p} d \mu\right)^{2 / p}
$$

for any $u \in \mathrm{H}^{1}\left(\mathbb{S}^{d}, d \mu\right)$ such that, using spherical coordinates,

$$
u\left(\theta, \varphi_{1}, \varphi_{2}, \ldots \varphi_{d-1}\right)=u\left(\pi-\theta, \varphi_{1}, \varphi_{2}, \ldots \varphi_{d-1}\right) \quad \forall\left(\theta, \varphi_{1}, \varphi_{2}, \ldots \varphi_{d-1}\right) \in[0, \pi] \times[0,2 \pi)^{d-1} .
$$

4.6. One more remark. The computation is exactly the same if $p \in(1,2)$ and we henceforth also prove the result in such a case. The case $p=1$ is the limit case corresponding to the Poincaré inequality

$$
\int_{-1}^{1}\left|f^{\prime}\right|^{2} d \nu_{d+2} \geq d\left(\int_{-1}^{1}|f|^{2} d \nu_{d}-\left|\int_{-1}^{1} f d \nu_{d}\right|^{2}\right)
$$

and arises as a straightforward consequence of the spectral properties of $\mathcal{L}$. The case $p=2$ is achieved as a limiting case. It gives rise to the logarithmic Sobolev inequality (see for instance [35]).

4.7. Limitation of the method. The limitation $p \leq 2^{\sharp}$ comes from the pointwise condition

$$
h:=\left|f^{\prime \prime}\right|^{2}+(p-1) \frac{d}{d+2} \frac{\left|f^{\prime}\right|^{4}}{f^{2}}-2(p-1) \frac{d-1}{d+2} \frac{\left|f^{\prime}\right|^{2} f^{\prime \prime}}{f} \geq 0 .
$$

Can we find special test functions $f$ such that this quantity can be made negative ? which are admissible, i.e. such that $h \nu^{2}$ is integrable? Notice that at $p=2^{\sharp}$, we have that $f(x)=|x|^{1-d}$ is such that $h \equiv 0$, but such a function, or functions obtained by slightly changing the exponent, are not admissible for larger values of $p$.

By proving that there is contraction of $\mathcal{I}$ along the flow, we look for a condition which is stronger than asking that there is contraction of $\mathcal{F}$ along the flow. It is therefore possible that the limitation $p \leq 2^{\sharp}$ is intrinsic to the method.

Acknowledgements. J.D. and M.J.E. were partially supported by ANR grants CBDif and NoNAP, and J.D. by the ECOS project C11E07 Functional inequalities, asymptotics and dynamics of fronts. M.K. was partially supported by Chilean research grants Fondecyt 1090103, Fondo Basal CMM-Chile, Project Anillo ACT-125 CAPDE. M.L. was supported in part by NSF grant DMS-0901304.

(c) 2012 by the authors. This paper may be reproduced, in its entirety, for non-commercial purposes. 


\section{REFERENCES}

[1] A. Arnold, J.-P. Bartier, And J. Dolbeault, Interpolation between logarithmic Sobolev and Poincaré inequalities, tech. rep., Ceremade no. 0528, 2005.

[2] — Interpolation between logarithmic Sobolev and Poincaré inequalities, Commun. Math. Sci., 5 (2007), pp. 971-979.

[3] A. Arnold and J. Dolbeault, Refined convex Sobolev inequalities, J. Funct. Anal., 225 (2005), pp. 337-351.

[4] A. Arnold, P. Markowich, G. Toscani, and A. Unterreiter, On convex Sobolev inequalities and the rate of convergence to equilibrium for Fokker-Planck type equations, Comm. Partial Differential Equations, 26 (2001), pp. 43100.

[5] T. Aubin, Problèmes isopérimétriques et espaces de Sobolev, J. Differential Geometry, 11 (1976), pp. 573-598.

[6] A. Baernstein, II And B. A. TAYLOR, Spherical rearrangements, subharmonic functions, and ${ }^{*}$-functions in $n$-space, Duke Math. J., 43 (1976), pp. 245-268.

[7] D. BAKRY, Une suite d'inégalités remarquables pour les opérateurs ultrasphériques, C. R. Acad. Sci. Paris Sér. I Math., 318 (1994), pp. 161-164.

[8] D. Bakry And A. Bentaleb, Extension of Bochner-Lichnérowicz formula on spheres, Ann. Fac. Sci. Toulouse Math. (6), 14 (2005), pp. 161-183.

[9] D. Bakry and M. Émery, Hypercontractivité de semi-groupes de diffusion, C. R. Acad. Sci. Paris Sér. I Math., 299 (1984), pp. 775-778.

[10] D. BAKRY AND M. Émery, Diffusions hypercontractives, in Séminaire de probabilités, XIX, 1983/84, vol. 1123 of Lecture Notes in Math., Springer, Berlin, 1985, pp. 177-206.

[11] W. Beckner, A generalized Poincaré inequality for Gaussian measures, Proc. Amer. Math. Soc., 105 (1989), pp. $397-$ 400 .

[12] - Sobolev inequalities, the Poisson semigroup, and analysis on the sphere $S^{n}$, Proc. Nat. Acad. Sci. U.S.A., 89 (1992), pp. 4816-4819.

[13] — Sharp Sobolev inequalities on the sphere and the Moser-Trudinger inequality, Ann. of Math. (2), 138 (1993), pp. 213-242.

[14] A. Bentaleb, Développement de la moyenne d'une fonction pour la mesure ultrasphérique, C. R. Acad. Sci. Paris Sér. I Math., 317 (1993), pp. 781-784.

[15] — Inégalité de Sobolev pour l'opérateur ultrasphérique, C. R. Acad. Sci. Paris Sér. I Math., 317 (1993), pp. 187-190.

[16] A. Bentaleb, Sur l'hypercontractivité des semi-groupes ultrasphériques, in Séminaire de Probabilités, XXXIII, vol. 1709 of Lecture Notes in Math., Springer, Berlin, 1999, pp. 410-414.

[17] A. Bentaleb, L'hypercontractivité des semi-groupes de Gegenbauer multidimensionnels-famille d'inégalités sur le cercle, Int. J. Math. Game Theory Algebra, 12 (2002), pp. 259-273.

[18] - Sur les fonctions extrémales des inégalités de Sobolev des opérateurs de diffusion, in Séminaire de Probabilités, XXXVI, vol. 1801 of Lecture Notes in Math., Springer, Berlin, 2003, pp. 230-250.

[19] A. Bentaleb and S. Fahlaoui, Integral inequalities related to the Tchebychev semigroup, Semigroup Forum, 79 (2009), pp. $473-479$.

[20] —, A family of integral inequalities on the circle $\mathbf{S}^{1}$, Proc. Japan Acad. Ser. A Math. Sci., 86 (2010), pp. 55-59.

[21] M. Berger, P. Gauduchon, and E. Mazet, Le spectre d'une variété riemannienne, Lecture Notes in Mathematics, Vol. 194, Springer-Verlag, Berlin, 1971.

[22] M.-F. Bidaut-VÉRON ANd L. VÉRON, Nonlinear elliptic equations on compact Riemannian manifolds and asymptotics of Emden equations, Invent. Math., 106 (1991), pp. 489-539.

[23] F. Bolley and I. Gentil, Phi-entropy inequalities and Fokker-Planck equations, in Progress in analysis and its applications, World Sci. Publ., Hackensack, NJ, 2010, pp. 463-469.

[24] — Phi-entropy inequalities for diffusion semigroups, J. Math. Pures Appl. (9), 93 (2010), pp. 449-473.

[25] F. Brock, A general rearrangement inequality à la Hardy-Littlewood, J. Inequal. Appl, 5 (2000), pp. 309-320.

[26] E. Carlen and M. Loss, Competing symmetries, the logarithmic HLS inequality and Onofri's inequality on $S^{n}$, Geom. Funct. Anal., 2 (1992), pp. 90-104.

[27] D. Chafä̈, Entropies, convexity, and functional inequalities: on $\Phi$-entropies and $\Phi$-Sobolev inequalities, J. Math. Kyoto Univ., 44 (2004), pp. 325-363.

[28] P. Funk, Beiträge zur Theorie der Kegelfunktionen., Math. Ann., 77 (1915), pp. 136-162.

[29] B. Gidas And J. Spruck, Global and local behavior of positive solutions of nonlinear elliptic equations, Comm. Pure Appl. Math., 34 (1981), pp. 525-598.

[30] L. Gross, Logarithmic Sobolev inequalities, Amer. J. Math., 97 (1975), pp. 1061-1083.

[31] E. Hebey, Nonlinear analysis on manifolds: Sobolev spaces and inequalities, vol. 5 of Courant Lecture Notes in Mathematics, New York University Courant Institute of Mathematical Sciences, New York, 1999.

[32] E. Hecke, Über orthogonal-invariante Integralgleichungen., Math. Ann., 78 (1917), pp. 398-404.

[33] R. Latala and K. Oleszkiewicz, Between Sobolev and Poincaré, in Geometric aspects of functional analysis, vol. 1745 of Lecture Notes in Math., Springer, Berlin, 2000, pp. 147-168.

[34] E. H. LieB, Sharp constants in the Hardy-Littlewood-Sobolev and related inequalities, Ann. of Math. (2), 118 (1983), pp. 349-374.

[35] C. E. Mueller and F. B. Weissler, Hypercontractivity for the heat semigroup for ultraspherical polynomials and on the n-sphere, J. Funct. Anal., 48 (1982), pp. 252-283.

[36] G. Rosen, Minimum value for $c$ in the Sobolev inequality $\phi^{3}\|\leq c \nabla \phi\|^{3}$, SIAM J. Appl. Math., 21 (1971), pp. $30-32$.

[37] G. Talenti, Best constant in Sobolev inequality, Ann. Mat. Pura Appl. (4), 110 (1976), pp. 353-372.

[38] F. B. Weissler, Logarithmic Sobolev inequalities and hypercontractive estimates on the circle, J. Funct. Anal., 37 (1980), pp. 218-234. 
J. Dolbeault: Ceremade, Université Paris-Dauphine, Place de lattre de Tassigny, 75775 Paris Cédex 16, FRANCE. E-mail address: dolbeaul@ceremade.dauphine.fr

M.J. Esteban: Ceremade, Université Paris-Dauphine, Place de Lattre de Tassigny, 75775 Paris Cédex 16, FRANCE. E-mail address: esteban@ceremade.dauphine.fr

M. Kowalczyk: Departamento de Ingeniería Matemática and Centro de Modelamiento Matemático (UMi 2807 CNRS), Universidad de Chile, Casilla 170 Correo 3, Santiago, Chile. E-mail address: kowalczy@dim.uchile.cl

M. Loss: Skiles Bullding, Georgia Institute of Technology, Atlanta GA 30332-0160, USA. E-mail address: loss@math.gatech.edu 